\title{
Proposal for Improvement of Information Transmission in OFDM Systems Using the CBEDE Methodology
}

\author{
Reinaldo Padilha \\ Yuzo Iano \\ Ana Carolina Borges Monteiro \\ Rangel Arthur
}

Padilha, Reinaldo, lano, Yuzo, Monteiro, Ana Carolina Borges and Arthur, Rangel; 2019. Proposal for Improvement of Information Transmission in OFDM Systems Using the CBEDE Methodology. SET INTERNATIONAL JOURNAL OF BROADCAST ENGINEERING. ISSN Print: 2446-9246 ISSN Online: 2446-9432. doi: 10.18580/setijbe.2019.6. Web Link: http://dx.doi.org/10.18580/setijbe.2019.6 


\title{
Proposal for Improvement of Information Transmission in OFDM Systems Using the CBEDE Methodology
}

\author{
Reinaldo Padilha, Yuzo Iano, Ana Carolina Borges Monteiro and Rangel Arthur
}

\begin{abstract}
In a world where people are increasingly connected, one of the key challenges is better quality data transmission, just as the increase in demand increases the need for attention to choose the best technology according to each transmission need, mainly by voice and video. The Orthogonal Frequency Division Multiplexing (OFDM) modulation technique has emerged as technological evolution, wherein a conventional transmission system, the symbols are sent in sequence through a single carrier, whose spectrum occupies the entire available frequency range, however OFDM consists in the parallel transmission of data in several subcarriers, with PSK modulation and with sub-carrier transmission rates as low as the number of them. Based on that, the present study implements a model based on discrete events applied to a broadcasting system, using the Simulink of the MATLAB software, aiming to improve the transmission of content, through the pre-coding process of bits applying discrete events in the signal before the modulation process. This proposal brings a different approach, in which the signal transmission on the channel is realized in the discrete domain with the implementation of discrete entities in the process of bit generation. The results show better computational performance related to memory utilization relative to the compression of the information, showing improvement of more than $22 \%$.
\end{abstract}

Index Terms - discrete events, OFDM, DPQSK, memory, simulation, bits.

R. Padilha is currently studying for a Ph.D.'s degree in Electrical Engineering, acting Laboratory of Visual Communications at the State University of Campinas (padilha@decom.fee.unicamp.br).

Y. Iano is teacher and coordinator of the Laboratory of Visual Communications at the State University of Campinas (yuzo@decom.fee.unicamp.br)

\section{INTRODUCTION}

In the area of telecommunications, new

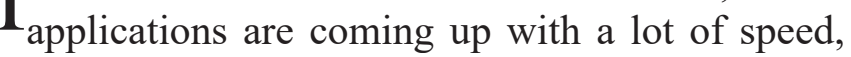
which requires a big compromise between the transmitted bit rate and the bandwidth, which can be seen in the current multimedia systems, bit rates vary from a few kbps (for voice), up to 20 Mbps, to HDTV (High Definition Television), the high definition television. Thus, when facing such challenges, the first point to consider is how to transmit a large number of bits/s, guaranteeing the quality of the service, that is, what modulation, as well as the best and most appropriate system, can meet, in the best way, the contradictory commitments (bit rate and bandwidth) [1].

A solution horizon that could be the use of equalization techniques in the receiver; however, there are practical difficulties with the way this real-time equalization operates at rates of the order of Mbps, and the use of compact and low-cost hardware. Therefore, a very promising technology that eliminates the need for complex equalizers is OFDM (Orthogonal Frequency Division Multiplexing), being a modulation technique that uses multiple carriers [2].

Nowadays the demand for bandwidth is increasing, especially for mobile devices, in that way OFDM is introduced as one of the solutions to enable bandwidth efficiency and robustness, because this technology is a basic building block for many of the current modulation schemes including 802.11 WLAN, 802.16 WiMAX, and 3GPP LTE, as well as others [2] [3].

The principle of OFDM technology is to divide the high data rate stream into parallel low rate data streams using Fast Fourier Transform (FFT), this

A. C. B. Monteiro is currently studying for a Ph.D.'s degree in Electrical Engineering, acting Laboratory of Visual Communications at the State University of Campinas (monteiro@decom.fee.unicamp.br).

R. Arthur is a teacher of Faculty of Technology (FT) of Unicamp, lecturer, and advisor to the Innovation Agency (Inova) of Unicamp (rangel@ft.unicamp.br). 
scheme is widely used in wireless communication system, such as IEEE 802.11 standard, cellular communication (eg, WiMAX and LTE Advanced) and digital broadcasting standard (e.g., DVB-T), and considering that OFDM technology is combined by modulation with more bits per symbol, thus increasing data transmission throughput [2] [4].

OFDM is a digital multi-carrier modulation scheme that extends the concept of single subcarrier modulation by using multiple subcarriers within the same single channel, i.e., is a multichannel modulation-based transmission system, also called a parallel or multiplexed transmission system, which then appears as an alternative to alleviate the problems of the serial system, which uses simple carrier modulation [5].

Rather than transmit a high-rate stream of data with a single subcarrier, OFDM makes use of a large number of closely spaced orthogonal subcarriers that are transmitted in parallel. Each subcarrier is modulated with a conventional digital modulation scheme (such as QPSK, 16QAM, DQPSK, as well as any others) at the low symbol rate. Generating a technological advantage of parallel or multi-carrier transmission, on the serial transmission, reducing the sensitivity of the system to channel delay spread, and therefore, interference between symbols [6] [7].

The technique of Discrete Event is focused on its use in the representation of a given system, which analyzes this as a sequence of operations performed through entities of certain types such as data packets, in this case for a telecommunications system. These entities are discrete items of interest in modeling and simulation with the discrete event technique, being their respective meaning depending on what is modeled and the type of system, in the same way, can have attributes that affect the way they are handled or changing the entity flows through the process [8] [9].

Discrete events are the results of actions occurring through the system, an event has the property of changing the system state, being these intentional actions, spontaneously controlled or with the verification of a condition. The technique has been used a lot in the modeling of concepts with a high level of abstraction, that is to say, patients in a hospital in context of a healthcare system, people in a queue, transaction systems for databases, communication protocols in telecommunication's systems, people in call centers, vehicles in intelligent transport systems, process control in control engineering, military equipment in defense systems, emails on a server, data packets being transmitted in communication system , among others [8] [9] [10].

Thus, in this research, the authors developed an OFDM transmission model for broadcasting using an AWGN (Additive White Gaussian Noise) channel with advanced modulation format DQPSK (Differential Quadrature Phase Shift Keying) in simulation environment [42], with the objective of to increase the transmission capacity of information content through the channel related to the lower memory consumption.

The proposal comes from the bit treatment with discrete events methodology, named CBEDE (Coded of Bits for Entities by means of Discrete Events), modeled in the step of bit generation, with the added differential of the use of discrete events applied in the physical layer of a transmission channel, being this a low-level of abstraction, reaching the second objective this research.

The present paper is organized as follows: Section 2 discusses traditional OFDM modeling, showing the modeling of AWGN transmission channel. Section 3 presents and presents the proposed framework of this paper, presenting the CBEDE methodology. Section 4 presents the results and, finally, in Section 5, the conclusions and the potential of the research are presented.

\section{TRADITIONAL MODEL}

The basic concept of OFDM is the property of multiple narrow-band channels, with the ability to send samples concurrently using multiples orthogonal sub-channels whereas in other techniques they are only wide-band channel sending the only sample using the entire band, OFDM is better due to the characteristic of multiple sub-channels (sub-carriers) carry samples sent at a lower rate, being almost same bandwidth with wide-band channel, and only some of the sub-channels are affected by interferers, and with the additional do not need guard bands [11].

OFDM is, therefore, a multiplexing scheme which divides the data stream from the narrowband data channel, sharing the bandwidth available, OFDM has subcarrier that orthogonality, unlike its predecessor Frequency Division Multiplexing (FDM) [11] [12].

This method is then based on the well-known FDM technique, wherein it different streams of information are mapped onto separate parallel channels, each of these channels being separated 
from the others by a frequency guard band thus reducing interference between adjacent channels. Since the OFDM scheme differs from traditional FDM in interrelated ways like multiple carriers (called subcarriers) carry the information stream, its subcarriers are orthogonal to each other, and a guard interval is added to each symbol to minimize the channel delay spread and intersymbol interference [13].

OFDM is a relatively new spectrally efficient digital modulation scheme employing multiple carriers that are mutually orthogonal to one another over a given time interval, which each carrier, consisting of a pair of sine wave and a cosine wave, is referred to as a subcarrier ', and the available transmission bandwidth is equally divided among the N sub-carriers. Each of them, upon data modulation, may be categorized as a narrowband modulated signal but the overall OFDM signal is a wideband signal for a moderate or large value of ' $\mathrm{N}$ '. As the modulation operation is carried out at its baseband level, and consequently the baseband modulated signal is translated in the frequency domain by frequency up-conversion to the required radio frequency band, as well as another desired type [5] [14].

In this way, the data bits are converted from serial to parallel and each subcarrier is modulated using phase or amplitude modulation, in which the frequency domain, multiple adjacent tones or subcarriers are independently modulated with complex data, this is called the symbol mapping, where each subcarrier is independently modulated. An interesting and particular practical feature of the OFDM modulation scheme consists of that shaping pulse is not necessary for the modulating signals due to a group of orthogonal carriers, when modulated by random pulse sequences, have sketched spectral characteristics [15].

Then, all the modulated signals are carried by OFDM carrier where they use IFFT module (performed on the frequency-domain subcarriers to produce the OFDM symbol in the time-domain) to create complex signal containing all subcarrier. The data parallel stream is converted to serial stream and real and image signal respectively are processed on Digital to Analog Converter. Then, they are quantized by Analog to Digital Converter $\mathrm{ADC}$ and the signal is calculated by a Fast Fourier Transform (FFT) module. The data symbols are then demodulated by symbol mapping block according to the modulation used, and the parallel data stream is converted into data serial to obtain the desired data [7] [15].
The importance of orthogonally of the OFDM signal is described as a set of closely spaced FDM subcarriers, wherein the frequency domain, each transmitted subcarrier results in a sync function with side lobes producing overlapping spectral between subcarriers, which has reducing properties of interference between a subcarrier and increase spectrum efficiency utilization [5] [16].

Resulting in subcarrier interference with the exception of orthogonally spaced frequencies, where the individual peaks of subcarriers all line up with the nulls of the other subcarriers, not interfering so with the system's ability to recover the original signal (the receiver correlates the incoming signal recovering the original set of bits sent) [5] [15] [16].

This modulation has another important issue with regard to the effect of the transmission channel, having high spectral efficiency, favored data transfer at high rates (typically beyond $1 \mathrm{Mbps}$ ) in wireless networks. In this way, the use of orthogonal subcarriers allows more subcarriers per bandwidth, which results in an increase in spectral efficiency, and prevents interference between overlapping carriers [5] [17].

OFDM has a wide range of applications in modern wireless digital transmission systems asynchronous digital subscriber line (ADSL), high-speed DSL, very high-speed DSL use OFDM for high-speed data transfer transmission, as well as Digital Audio Broadcasting (DAB) and Digital Video Broadcasting (DVB), still considering IEEE 802.11a, IEEE 802.11g and HYPERLAN2 wireless Local Area Network (WLAN) standards include OFDM for supporting higher bit rates and IEEE 802.16 Wireless Metropolitan Area Network (MAN) standard [18].

The AWGN channel is a practical and efficient model of communication system widely used related to its simplicity and mathematical treatment, implying in a large set of physical channels, which introduces in the transmitted signals a noise modeled statistically as a white Gaussian additive process [19].

In the context of wireless communications, the main source of thermal noise is the addition of random signals arising from the vibration of atoms in the receiver electronics, where the AWGN has properties of statistically characterizing these random radio noise present in the signal. Still taking into consideration that systems operating in AWGN conditions can be exemplified as space communications with highly directional antennas and some point-to-point microwave links [20] [21] [22]. 
The modulation format DQPSK (Differential Quadrature Phase Shift Keying) is widely used in satellite broadcasting, for Digital Video and Radio Broadcasting, having a wide scope of performance in various cellular wireless standards like GSM, CDMA, LTE, 802.11 WLAN, 802.16 fixed and mobile WiMAX, as well as CABLE TV applications. In the same way, it has properties for higher bit rates of HD video and a high satellite bandwidth [23] [24].

In DQPSK, each set of bits is represented by a symbol, causing a determined phase variation in the carrier signal, the bits for the data symbols are determined by the phase change of the previous symbol, there were four possible states $0, \pi,+\pi / 4$, $-\pi / 4$, where each symbol represents two bits of information. The division of the binary pattern is equal to QPSK (Quadrature Phase Shift Keying), except that a bit string is shifted in phase with respect to $\pi / 4$ or $\pi / 2$ depending on the system being implemented. That is, there are 8 ideal state positions in the DQPSK constellation, being ideal state positions for symbols alternating between the four states of 45 degrees and four states on the axis, due to this alternation, the ideal trajectory between symbols never crosses zero [23] [24].

This session is presented to broadcasting OFDM system modeled with an AWGN channel with DQPSK modulation. Thus, was used the Simulink simulation environment of the MATLAB ${ }^{\circledR}$ software in its version 8.3 of 64 bits (2014a).

In a digitally implemented OFDM system, the input bits are generated frame-based, 16-ary random integers with 960 samples/frame, grouped and mapped to source data symbols that are a complex number, after are modulated with DQPSK modulation, then shaping of OFDM symbols, before 960 samples/frame and are now converted into a $48 \times 20$ array, creating an efficient FFT size, and thus appends zeroes to increase the column size to 64, which modifies the indices of the array for input to the Inverse Fast Fourier transform (IFFT). With the column size set at 64, the OFDM modulation is implemented using a 64point IFFT, and a gain is applied which applies a normalization of $1 / \sqrt{ } 64=1 / 8$ [5] [25].

And then the Cyclic Prefix, whose parameters are basically added to appends 16 symbols for the cyclic prefix and expands the output array to $80 \times 20$ are added. Passing through a digital-toanalog conversion, which modifies the $80 \times 20$ array to a single dimension for input to the Saleh model, which characterizes the power amplifier [26] [27] [28].

This model is operated with $\mathrm{AM} / \mathrm{AM}=\left[\begin{array}{ll}1 & 0\end{array}\right]$ and $\mathrm{AM} / \mathrm{PM}=\left[\begin{array}{ll}0 & 0\end{array}\right]$, thereby forcing the OFDM signal to remain within the linear region of the power amplifier, causing the OFDM signal to remain in the nonlinear region of the power amplifier [26] [27] [28].

The setting of the AWGN channel follows a signal-to-noise ratio of $15 \mathrm{~dB}$, initial seed of 67 , power input signal of 1 watt and sample time of 1 second. And then the signal again is converted into a single dimensional vector back to an $80 \times 20$ array, analog to digital conversion, removing the Cyclic Prefix, following the gain normalization at $1 / \sqrt{ } 64=1 / 8$, and a 64 -point FFT is performed, making the way back to the original state of the signal, again producing the frame-based signal, removing the inserted zeros, producing the $48 \times 20$ array [26] [27] [29].

Performing a conversion again on the signal to which this signal with $48 \times 20$ array back to the 960-sample frame for insertion into the DQPSK demodulator [30].

What this complex process described above accomplishes is that the generated bits, as well as the OFDM symbols, are treated by the transmitter as though they are in the frequency domain and are the inputs to an IFFT that transforms the data into the time-domain [5] [31].

The IFFT takes in $\mathrm{N}$ source symbols at a time where $\mathrm{N}$ is the number of subcarriers in the system. Each of these $\mathrm{N}$ input symbols has a symbol of T seconds. Recall that the output of the IFFT is N orthogonal sinusoids. These orthogonal sinusoids each have a different frequency and the lowest frequency is DC [5] [31].

The IFFT output is the summation of all $\mathrm{N}$ sinusoids. Thus, the IFFT provides a simple way to modulate data onto $\mathrm{N}$ orthogonal subcarriers. The $\mathrm{N}$ output samples from the IFFT make up a single OFDM symbol. After some additional processing, the time-domain signal that results from the IFFT is transmitted across the AWGN channel. At the receiver, an FFT is used to process the received signal and bring it into the frequency domain which is used to recover the original data bits, as shown in Figure 01 [5] [32]. 


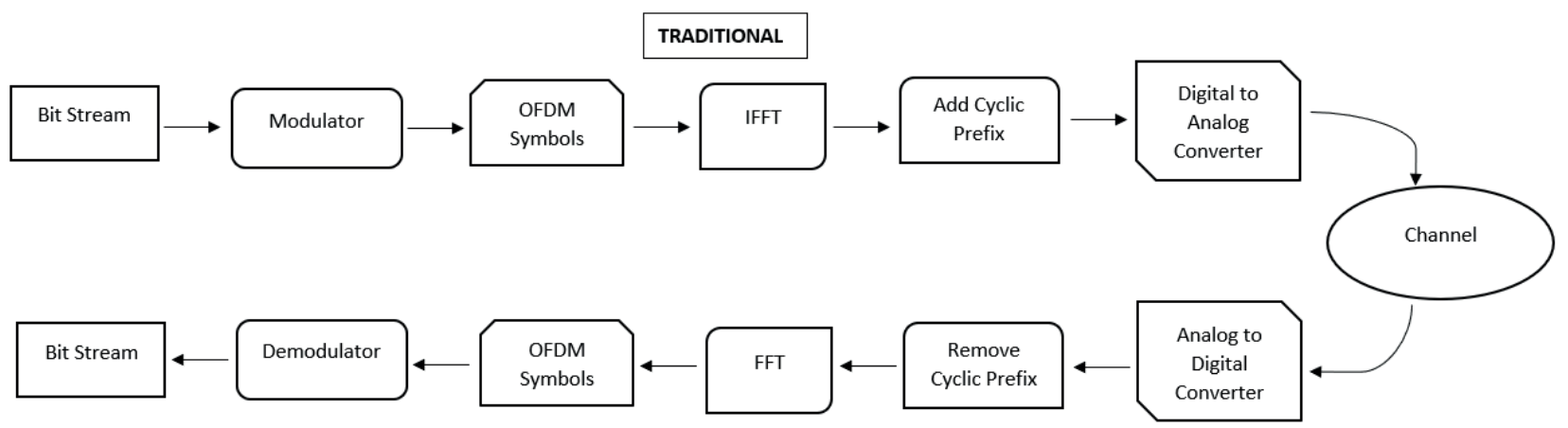

Figure 01 - Traditional Model

\section{PROPOSAL}

The modeling of the pre-coding process according to the discrete events is similar to that shown in the previous section. Differentiating that in this model, was added the discrete events of pre-coding with discrete events.

In this way, discrete entities were generated in the bit generation process, corresponding to bits 0 and 1 , then a conversion of an event-based data signal to a time-based data signal is performed, where these bits are maintained in accordance with the discrete domain of interest, the value of the signal before the conversion being identical to that of the converted signal.

Both time-based signals and event-based signals will be in the time domain.

A Zero-Order Hold $(\mathrm{ZOH})$ is responsible for defining the sampling in a practical sense, being used for discrete samples at regular intervals, affecting the final effect of the conversion of the signal to the time domain, causing its reconstruction and maintaining each sample value for a specific time interval and identical to the original.

The differential of this research is in the use of discrete events applied in such low-level of abstraction, being the bit generation.
And after applying the technique in the step of generating bits as discrete entities, the signal flow is identical to that shown in the previous section, passing through the respective OFDM steps, being modulated in DQPSK, passing through the AWGN channel, and then the signal is demodulated.

The model presented in Figure 02, incorporates the traditional modeling with the proposed methodology, as well as highlights the part modeled according to the approach of discrete events, in blue, as previously described.

In Figure 03, in the simulation environment 10000 seconds were used, where for the comparative criterion the flows of transmission of the modulated signal in DQPSK signal related to OFDM with the proposal (below) and OFDM traditional model (top) were placed, noting that both methodologies generated the same result.

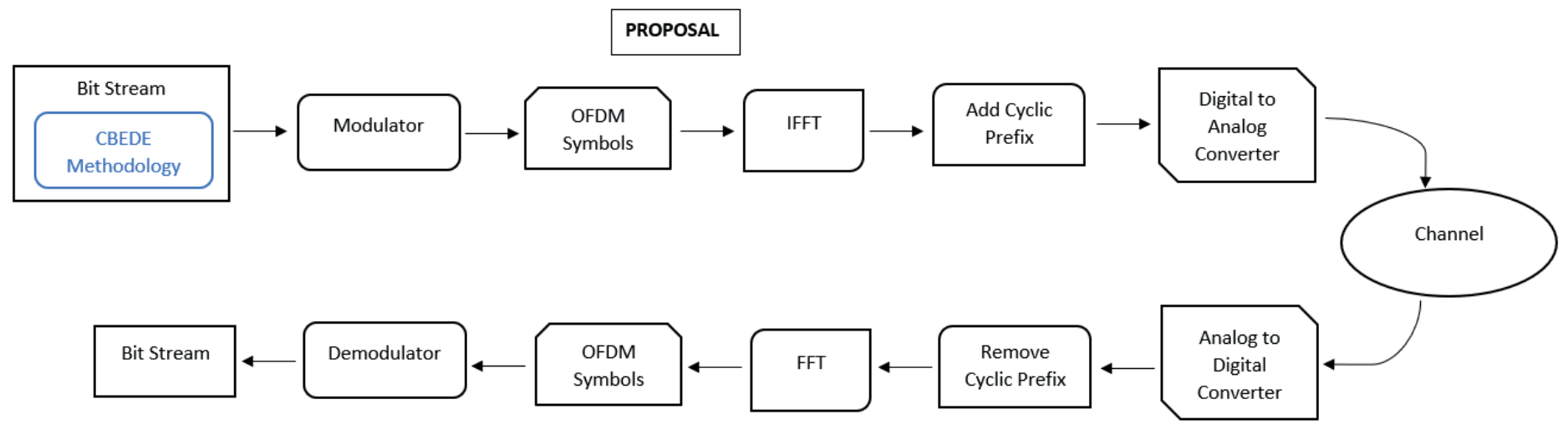

Figure 02 - Model with the proposal 


\section{RESULTS}
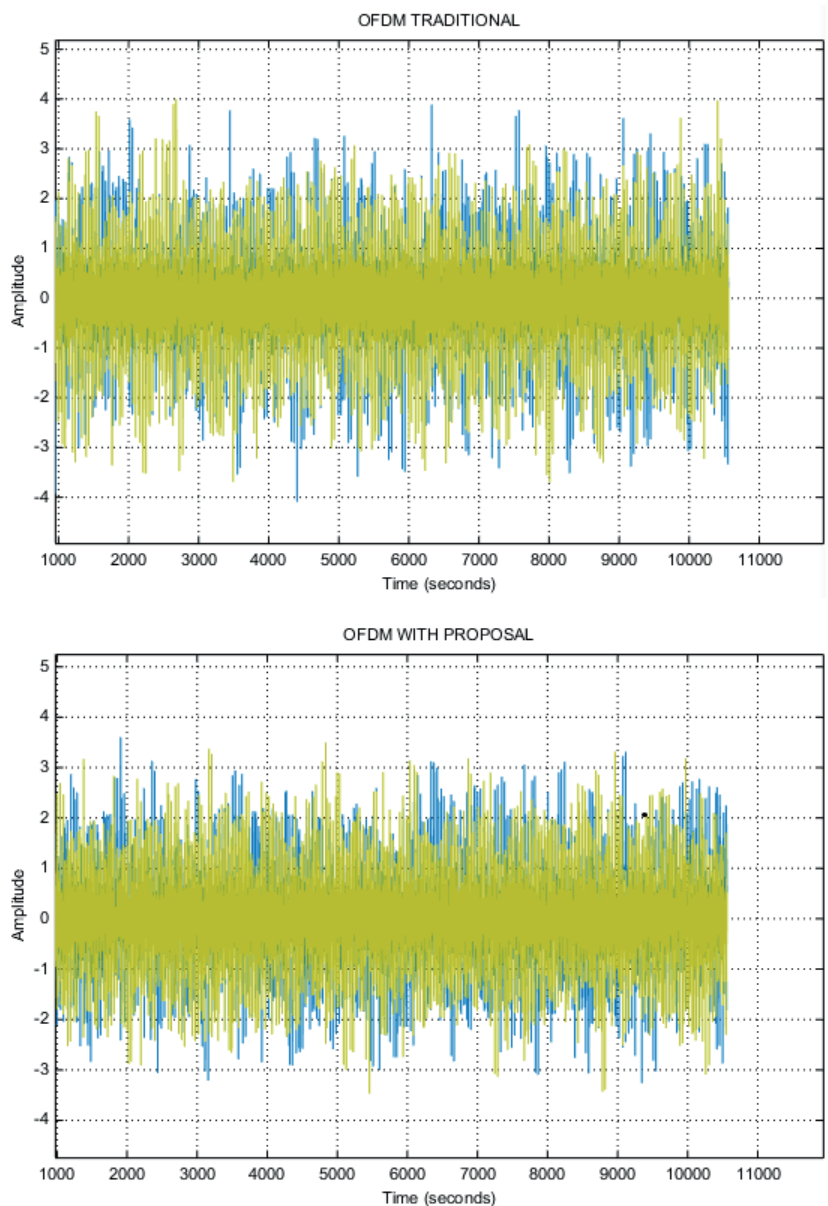

Figure 03 -Transmission Flow DQPSK OFDM

For the same comparative purpose, the Constellation Diagram was used to view the constellation of the modulated digital signal OFDM also being useful for comparing the performance of one system with another.

In Figure 04 is shown the results for visualization of the constellations with $15 \mathrm{~dB}$, according to signal related to OFDM with the proposal (right) and OFDM traditional model (left).
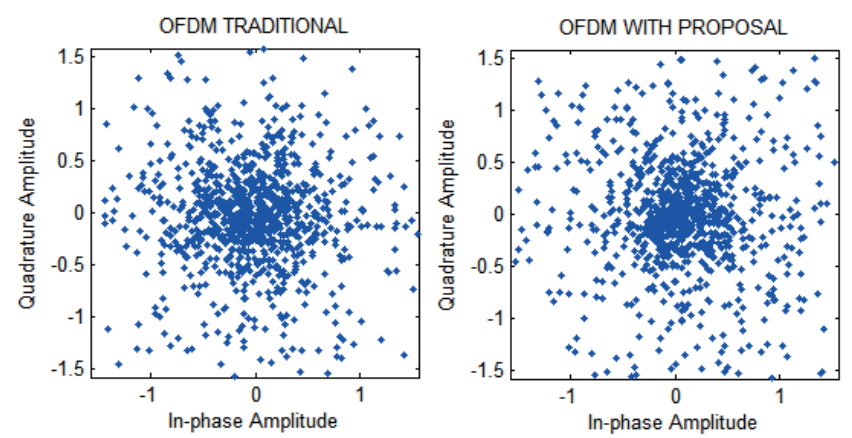

Figure 04 - DQPSK OFDM Constellations
In this section, the results will be presented in relation to the evaluations of memory consumption of the models according to the studied techniques, modeled and presented in the previous session. Also, it is presented the performance comparison between them, simulated on a physical machine with hardware configuration, being an Intel Core i3 processor and 4GB RAM.

The "sldiagnostics" function displays information about the modeling system in Simulink, being responsible for calculating the sum of all the memory consumption processes used in the model in simulation, by the ProcessMemUsage parameter, which counts the amount of memory utilized in each phase of the model, during the entire simulation, displaying the total amount in MB [33].

In the same way, it was analyzed the first simulation of both models, being observed the first simulation regarding their memory consumption [33], having the comparative of better performance as shown in TABLE $I$ and related with the Figure 05, with respect to them and that it is important to be in the first simulation that the variables are allocated, and the memory reserved for the execution of the model

TABLE I. COMPUTATIONAL IMPROVEMENT

\begin{tabular}{|c|c|c|}
\hline \multicolumn{3}{|c|}{ Memory Consumption } \\
\hline & traditional & proposal \\
\hline $\begin{array}{c}\text { Broadcasting DQPSK } \\
\text { OFDM }\end{array}$ & 60,3672 & 49,3359 \\
\hline
\end{tabular}

In this way, it can be understood that in a transmission channel including the proposal and in another one only traditional methodology, where it is transmitted the same information content (quantity of bits), without any loss (signal and constellation). The results related to the memory consumption of the proposal are relative to the compression of the information [33], as shown in TABLE I and related with Figure 05. 


\section{OFDM Memory Consumption (i3)}

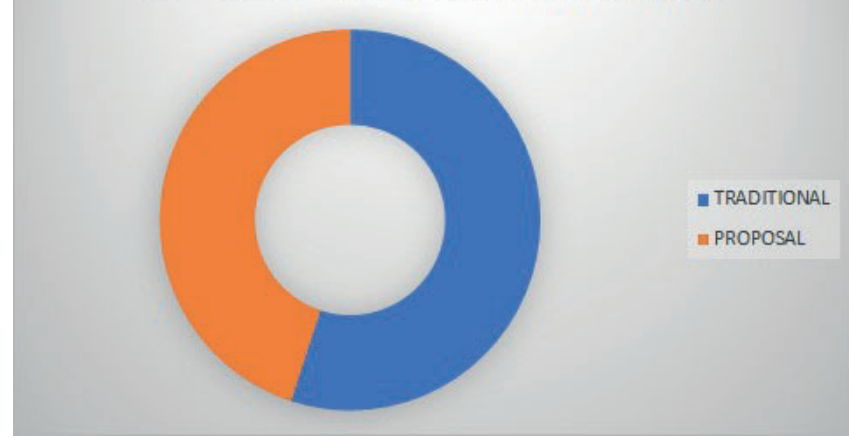

Figure 05 - OFDM First Memory Consumption

\section{CONCLUSIONS}

OFDM is a modulation and data transmission technique that uses its bandwidth divided into multiple orthogonal carriers, called subcarriers, for modulation, using the frequency spectrum with advanced techniques, in which it allows high data rate transmissions without problems with the ISI, by the use of orthogonality between the carriers, unlike the single carrier system, when frequency selective fading occurs only the symbols transmitted on the carriers affected by fading are lost. These subcarriers are called orthogonal because they do not have frequency overlap, thus not interfering with each other. It has a basic principle the conversion of a serial data stream of high transmission rate into multiple parallel substreams of the low transmission rate. Its main advantage over single carrier techniques is that it can achieve the same transfer rate due to the parallelism of low rate subcarriers with greater resistance to poor conditions of the medium such as high frequency attenuation, inter- symbol, interference caused by multiple paths (common in wireless networks, due to reflection).

Thus, the use of discrete events applied in a low-level of abstraction such as the bit, in generation phase in a broadcasting system which uses OFDM, was the differential of this research since it does not apply discrete events in this way. Where evaluating the results, it is shown that the simulation models of broadcasting systems take a different approach from what is normally done, a proposal containing a concept of a technique naturally applied at higher levels, in a lower abstraction level, in bits in the transmission of a channel, provides positive results with a $22.36 \%$ improvement to a naturally powerful technique (OFDM), which strengthens it even more.

Although taking into account that an extension of the results of this research, the compression of the information, strongly affects similar methods performed in higher layers, for example, format types such as HEVC, MPEG-4, AVC/H.264, as well the others, can improve them even more since this proposal acts on the bits.

\section{REFERENCES}

[1] Dunlop, John. Telecommunications engineering. Routledge, 2017.

[2] Abdoli, Javad, Ming Jia, and Jianglei Ma. "Filtered OFDM: A new waveform for future wireless systems." 2015 IEEE 16th International Workshop on Signal Processing Advances in Wireless Communications (SPAWC). IEEE, 2015.

[3] Rahman, MuhibUr, et al. "Bandwidth enhancement and frequency scanning array antenna using novel UWB filter integration technique for OFDM UWB radar applications in wireless vital signs monitoring." Sensors 18.9 (2018): 3155. [4] Wang, Yanyan, et al. "An enhanced OFDM for broadcasting systems using uniform subband division and multistage interpolator." 2017 IEEE International Symposium on Broadband Multimedia Systems and Broadcasting (BMSB). IEEE, 2017.

[5] Galande, N., and A. Shah. "Concepts and Brief Survey of Conventional and wavelet OFDM." International Journal of Science, Engineering and Technology Research 5.1 (2016): 149-152.

[6] Yeh, Chien-Hung, et al. "Utilization of multi-band OFDM modulation to increase traffic rate of phosphor-LED wireless VLC." Optics express 23.2 (2015): 1133-1138.

[7] Mao, Tianqi, et al. "Dual-mode index modulation aided OFDM." IEEE Access 5 (2016): 50-60.

[8] Brito, Thiago Barros, Edson Felipe Capovilla Trevisan, and Rui Carlos Botter. "A conceptual comparison between discrete and continuous simulation to motivate the hybrid simulation methodology." Proceedings of the Winter Simulation Conference. Winter Simulation Conference, 2011.

[9] Chahal, Kirandeep, and Tillal Eldabi. "A multiperspective comparison for selection between system dynamics and discrete event simulation." International Journal of Business Information Systems 6.1 (2010): 4-17.

[10] Cassandras, C. G., \& Lafortune, S. (2009). Introduction to discrete event systems. Springer Science \& Business Media.

[11] Zeng, Yong, and Rui Zhang. "Millimeter wave MIMO with lens antenna array: A new path division multiplexing paradigm." IEEE Transactions on Communications 64.4 (2016): 1557-1571.

[12] Bandy, William R., Yuri Okunev, and Wayne E. Shanks. "Data Transmission via Wide Band Acoustic Channels." U.S. Patent Application No. 14/948,764.

[13] Bhattacharya, Ansuman, et al. "Multimedia channel allocation in cognitive radio networks using FDM-FDMA and OFDM-FDMA." arXiv preprint arXiv:1603.03938 (2016).

[14] Bodinier, Quentin, Faouzi Bader, and Jacques Palicot. "Modeling interference between OFDM/OQAM and CPOFDM: Limitations of the PSD-based model." 2016 23rd International Conference on Telecommunications (ICT). IEEE, 2016. 
[15] Wen, Miaowen, et al. "On the achievable rate of OFDM with index modulation." IEEE Transactions on Signal Processing 64.8 (2015): 1919-1932.

[16] Saied, Osama, et al. "Single carrier optical FDM in visible light communication." 2016 10th International Symposium on Communication Systems, Networks and Digital Signal Processing (CSNDSP). IEEE, 2016.

[17] Muslimin, J., et al. "Experimental Studies on the Effect of Antenna Orientations to the Performance of OFDMbased System." International Journal of Electrical and Computer Engineering 8.4 (2018): 2588.

[18] Kumari, Shailly, and Rajesh Mehra. "Selective Mapping and Partial Transmit Sequence Based PAPR Reduction for OFDM Applications." IOSR Journal of VLSI and Signal Processing 6.6 (2016): 70-76.

[19] Rama Krishna, A., Chakravarthy, A. S. N. \& Sastry, A. S. C. S. (2016). Variable Modulation Schemes for AWGN Channel based Device to Device Communication. Indian Journal of Science and Technology, Vol 9(20), DOI: 10.17485 / ijst / 2016 / v9i20 / 89973.

[20] Bossert, M. (1999). Channel coding for telecommunications. John Wiley \& Sons, Inc..

[21] Lakshmanan, M. K., \& Nikookar, H. (2006). A review of wavelets for digital wireless communication. Wireless Personal Communications, 37(3-4), 387-420.

[22] Barnela, M., \& Kumar, D. S. (2014). Digital modulation schemes employed in wireless communication: A literature review. International Journal of Wired and Wireless Communications, 2(2), 15-21.

[23] Ling, S. O. A., Zen, H., Othman, A. K. B. H., Adnan, M., \& Bello, O. (2017). A Review on Cooperative Diversity Techniques Bypassing Channel Estimation. arXiv preprint arXiv:1711.10643.

[24] Tozer, Edwin Paul J. Broadcast engineer's reference book. Focal Press, 2012

[25] Fukuda, Rafael Masashi, and Taufik Abrão. "OFDM System Implementation in DSP Platform TMS320C6678." Journal of Computer and Communications 4.11 (2016): 26. [26] Xia, Xiang-Gen, Tianxian Zhang, and Lingjiang Kong. "MIMO OFDM radar IRCI free range reconstruction with sufficient cyclic prefix." IEEE Transactions on Aerospace and Electronic Systems 51.3 (2015): 2276-2293.

[27] Nagashima, T., et al. "Cyclic prefix insertion for alloptical fractional OFDM." 2015 International Conference on Photonics in Switching (PS). IEEE, 2015.

[28] Venkatesan, Sivarama, and Reinaldo A. Valenzuela. "OFDM for 5G: Cyclic prefix versus zero postfix, and filtering versus windowing." 2016 IEEE International Conference on Communications (ICC). IEEE, 2016.

[29] Zhao, Lu, Juan Montojo, and Christian Pietsch. "Hybrid waveform design combining OFDM and cyclic prefix based single carrier for millimeter-wave wireless communication." U.S. Patent No. 9,960,938. 1 May 2018.

[30] Abdoli, Javad, Ming Jia, and Jianglei Ma. "Filtered OFDM: A new waveform for future wireless systems." 2015 IEEE 16th International Workshop on Signal Processing Advances in Wireless Communications (SPAWC). IEEE, 2015.

[31] Galande, N., and A. Shah. "Concepts and Brief Survey of Conventional and wavelet OFDM." International Journal of Science, Engineering and Technology Research 5.1 (2016): 149-152.

[32] Heiskala, Juha, and John Terry Ph D. OFDM wireless LANs: A theoretical and practical guide. Sams, 2001.
[33] Padilha, Reinaldo França. "Proposta de um método complementar de compressão de dados por meio da metodologia de eventos discretos aplicada em um baixo nível de abstração= Proposal of a complementary method of data compression by discrete event methodology applied at a low level of abstraction." (2018).

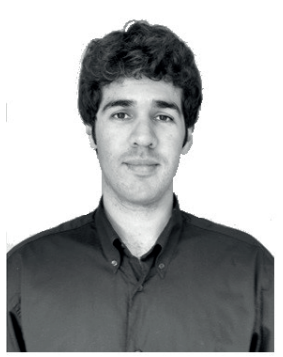

Reinaldo Padilha. Graduated in Computer Engineering (University Regional Center of Espírito Santo de Pinhal - 2014). Currently is a Ph.D. Candidate by Department of Communications (DECOM), Faculty of Electrical and Computer Engineering (FEEC) at State University of Campinas (UNICAMP), and a researcher at the Laboratory of Visual Communications (LCV). He also is currently Proceedings Chair of the Brazilian Symposium on Technology (BTSym). Has interest and affinity in the area of technological and scientific research as well as knowledge in programming and development in $\mathrm{C} / \mathrm{C}++$, Java and .NET languages. The main topics of interest are Simulation, Operating Systems, Software Engineering, Wireless and Network, Internet of Things, Broadcasting and Telecommunications Systems.

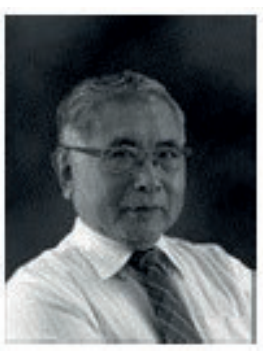

Prof ${ }^{\text {o }}$ Yuzo Iano. BS (1972) Master's degree (1974) and a Ph.D. degree (1986) in Electrical Engineering from the State University of Campinas, Brazil. Since then he has been working in the technological production field, with 1 patent granted, 8 patent applications filed, and 36 projects completed with research and development agencies. Successfully supervised 29 doctoral theses, 49 master's dissertations, 74 undergraduate and 48 scientific initiation works. He has participated in 100 master's examination boards, 50 doctoral degrees, author of 2 books and more than 250 published articles. He is currently Professor at the State University of Campinas, Brazil, Editor-in-Chief of the SET International Journal of Broadcast Engineering and General Chair of the Brazilian Symposium on Technology (BTSym). He has experience in Electrical Engineering, with knowledge in Telecommunications, Electronics and Information Technology, mainly in the field of audio-visual communications and data.

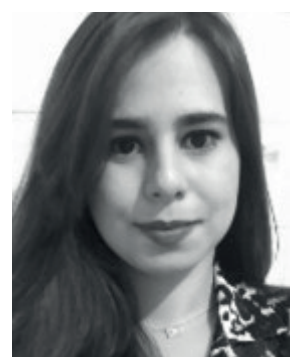

Ana Carolina Borges Monteiro. Graduated in Biomedicine from Centro Universitário Amparense UNIFIA (2015). Currently is a Ph.D. Candidate by Department of Communications (DECOM), Faculty of Electrical and Computer Engineering (FEEC) at State University of Campinas

(UNICAMP), and a researcher at the Laboratory of Visual Communications (LCV). She also is currently Registration Chair of the Brazilian Symposium on Technology (BTSym). Has expertise in the areas of Clinical Analysis 
and digital image processing through MATLAB software. This knowledge was acquired through the realization of research projects and internship in municipal hospital, as also experience in the revision of scientific works by acting as a reviewer in congresses.

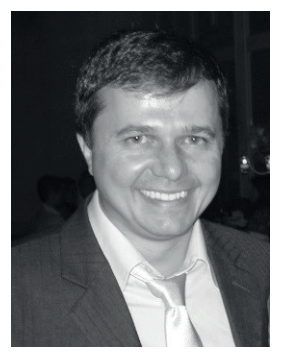

Rangel Arthur He holds a degree in Electrical Engineering from the Paulista State University Júlio de Mesquita Filho (1999), a Master's degree in Electrical Engineering (2002) and a $\mathrm{PhD}$ in Electrical Engineering (2007) from the State University of Campinas. Over the years from 2011 to 2014 he was Coordinator and Associate Coordinator of Technology Courses in Telecommunication Systems and Telecommunication Engineering of FT, which was created in its management. From 2015 to 2016 he was Associate Director of the Technology (FT) of Unicamp. He is currently lecturer and advisor to the Innovation Agency (Inova) of Unicamp. He has experience in the area of Electrical Engineering, with emphasis on Telecommunications Systems, working mainly on the following topics: computer vision, embedded systems and control systems. 